\title{
Reaction time to electrocutaneous onset and offset stimulation
}

THOMAS G. STICHT AND EMERSON FOULKE 2

UNIVERSITY OF LOUISVILLE

Reaction times were obtained from two $S$ s to the onset (beginning) and offset (cessation) of $70 \mathrm{cps} A C$ electrocutaneous stimuli of three sensation levels: low, medium and high. The results indicated that onset was faster than offset RTs at all three intensity levels.

In 1915, Woodrow found that reaction times (RTs) were faster to the onset of an alternating current than to the offset of the current. He reported that, following the offset of his stimuli, sensations of "after-tingling" occurred. He suggested that the latter phenomenon may have detracted from the detection of the offset of the stimulus, which would have resulted in longer RTs.

Woodrow's work was, by his own declaration, only of a cursory and crude nature. Because of the importance of understanding the basic nature of an electrocutaneous stimulation in the development of an electrocutaneous communication code, a project with which the authors are currently concerned (cf., Foulke, 1964), it was decided to reinvestigate the onset-offset problem confronted by Woodrow. In addition to the use of electronics equipment which permitted vastly improved control of the stimulus than that available to Woodrow in 1915, the present study differed from Woodrow's in that three stimulus intensities, ranging from very weak to strong, were used.

\section{Method}

Subjects were two male graduate students who were well practiced (three $h r$.-over 450 reactions) in reacting to electrocutaneous stimuli like those used in the present study.

A Hewlett-Packard audio oscillator (model 201CR) provided a $70 \mathrm{cps}$ alternating signal. The ouput of the oscillator was channeled through an attenuator (Daven Company, type 7717) to a Grayson-Stadler model 829D electronic switch. The ouput of the electronic switch was coupled to a 75 watt MeIntosh audio amplifier. The amplifier impedance was matched to S's impedance by a United Transformer, type L5-12. A 100K-ohm potentiometer (General Radio type 978-R) and a $100 \mathrm{~K}-\mathrm{ohm}$ fixed resistor were connected in series with $S$ in order to limit changes in stimulus current due to fluctuations in S's resistance. A $100 \mathrm{~K}-0 \mathrm{hm}$ precision resistor was also connected in series with $S$ and stimulus current was determined by measuring the voltage drop across this resistor with a Ballantine AC voltmeter (model $300 \mathrm{E}$ ). S's active electrode was a circular stainless steel disc ( $5 / 8$ in. diameter). The inactive electrode was a $3-1 / 2$ by $2-1 / 2$ in. stainless steel plate which covered the breadth of S's palm.
Stimulus intensity was adjusted by reference to the voltmeter. Stimulus foreperiods were controlled by means of a Hunter model $100 \mathrm{~B}$ decade interval timer which was connected to the external control curcuit of the electronic switch. Reaction times were measured with a Hunter Klockounter.

A single-pole, double-throw toggle switch, connected between the Hunter timer and the electronic switch, permitted two modes of stimulus presentation. With the toggle switch in one position, the signal came on when the Hunter timer was first activated and stayed on until the end of the interval set on the timer, at which time the electronic switch was triggered and the signal turned off. S reacted, under these conditions, to the offset of the signal. With the toggle switch in its other position, the electronic switch turned the signal on at the end of the interval set on the Hunter timer. In this case $S$ reacted to the onset of the signal.

Ss were seated in an Industrial Acoustics Company model 400 sound-deadened booth. To obtain a reaction, $\mathrm{E}$ set the foreperiod (which was varied randomly from 1.5 to $4.0 \mathrm{sec}$. in steps of 0.1 sec. to prevent synchronization of responses) on the Hunter timer and threw the toggle switch to one of the positions described above. Then he signalled $S$ that all was ready. $S$ placed the first phalange of his left index finger on the active electrode and rested his left palm on the inactive electrode. He produced his own stimulus by depressing a footswitch. The latter closed the Hunter timer circuit, thus beginning the foreperiod. At the end of the foreperiod, the timer triggered the electronic switch and, at the same time, closed the Klockounter circuit. In series with the Klockounter circuit was S's response (telegraph) key. S held the key closed until he felt the reaction signal. He then released the key which broke the Klockounter circuit. When the Klockounter circuit was broken, the time from the intiation of the stimulus until the circuit was broken was registered on the Klockounter. This time was recorded by $\mathrm{E}$ as S's reaction time.

Three intensities were used. For S S.Z., these were 440,560 and $700 \mu \mathrm{A}$, and for S H.T., they were 480 , 570 and $720 \mu \mathrm{A}$. There were six experimental conditions (onset and offset stimuli at three intensities). During each session 25 RTs (taken in blocks of five per condition) were obtained for each stimulus combination. Hence, 150 RTs were taken each experimental session. There were two sessions, giving a total of 50 RTs per condition. Stimulus combinations were counterbalanced to preclude order effects. 
Table 1. Means and Standard Deviations of the Reaction Times for Two Subjects to the Onset and Offset of $A$. C. Electrocutaneous Stimuli at Three Levels of Intensity (see text for intensity in $\mu \mathrm{A}$ ).

\begin{tabular}{llrrrrrr} 
& & \multicolumn{6}{c}{$\begin{array}{c}\text { Intensity } \\
\text { Medium }\end{array}$} \\
\cline { 3 - 8 } Ss & Score & \multicolumn{2}{c}{ Low } & \multicolumn{2}{c}{ High } \\
\cline { 3 - 8 } & & Onset & Offset & Onset & Offset & Onset & Offset \\
\hline S.Z & Mean & 214 & 352 & 171 & 237 & 162 & 227 \\
& S.D. & 56 & 133 & 25 & 33 & 14 & 23 \\
H.T. & Mean & 347 & 476 & 257 & 297 & 214 & 254 \\
& S.D. & 63 & 103 & 27 & 47 & 29 & 18 \\
\hline
\end{tabular}

Results

The median RT for each block of five trials was computed, and the mean of these 10 medians for each mode by intensity combination for each $\mathrm{S}$ was obtained. Table 1 presents these data, along with the corresponding standard deviations. Figure 1 presents these data graphically with mean RT (in msec.) on the ordinate and intensity ( $I$ ) on the abscissa. It is apparent from the figure that offset RTs are in all cases longer than onset RTs.

The Wilcoxon matched-pairs signed ranks test (Siegel, 1956, pp. 75-83) was performed with the 10 median onset and offset RTs obtained under each intensity for each $S$, and confirmed the differences indicated in Fig. 1 (in all cases $p<0.01$ ). The intensity variable was tested for significance using the Friedman two-way analysis of variance performed on the mean data presented in Table 1. This analysis indicated that the changes in both onset and offset RTs, as a function of intensity, were significant $(p<0.005)$. Both onset and offset RTs decrease as intensity increases. The difference between the offset and onset RTs is greatest

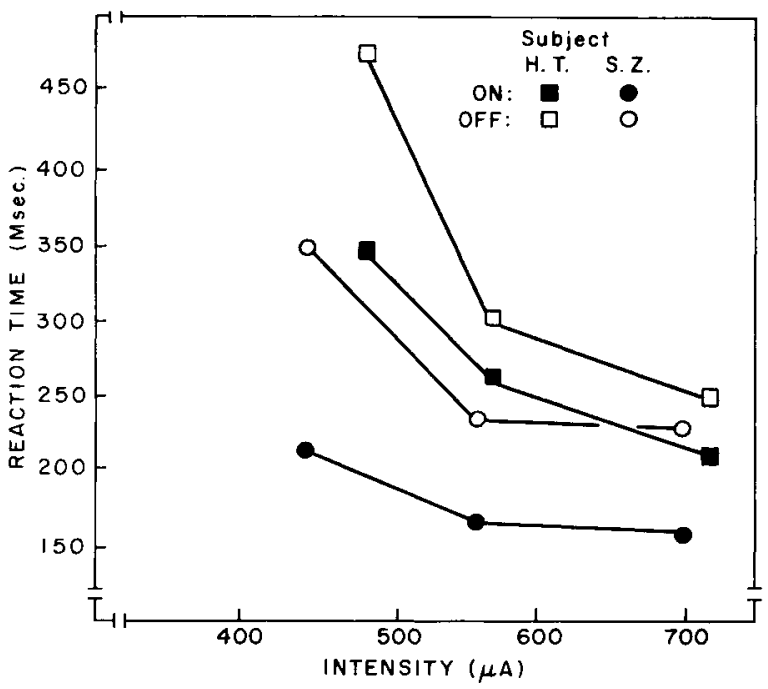

Fig. 1. Mean RTs for two Ss to onset and offset of electrocutaneous stimuli plotted against stimulus intensity in microamperes $(\mu \mathrm{A})$. at the lowest intensity, but does not appear to change much between the middle and strongest intensity. Visual inspection suggests that the rate of increase in RT as the intensity is decreased is greater for offset than for onset RTs.

\section{Discussion}

These results confirm those of Woodrow (1915) in demonstrating that the RT to electrocutaneous stimulation is faster to the onset than to the cessation of stimulation. Woodrow attributed this to "after-tingling" set up in the fingers by his stimuli, which thus acted as a mask for the stimulus offset. In the present study, no obvious "after-tingling" occurred. Ss reported that there was a tendency for the stimulus to "adapt-out" (i.e., fading of sensation) with continued application. This was most obvious with the weakest intensity. Thus, the subjective intensity of the stimulus was less at the offset than at the onset-a fact indexed by the RT data (both the subjective reports and RT findings were subsequently replicated with a third $S$, the second author).

Data of others suggest that electrical stimuli stimulate peripheral nerves rather than (or in addition to) specialized receptor endings (cf., Hawkes, 1960, p. 53). It is known that such nerves show adaptation to constant current stimuli (Adrian, 1928, pp. 68-69). By adaptation is meant a gradual decrease in the total number of neural impulses propagated by a peripheral nerve with continued constant current stimulation. The results of the present study suggest that with the onset of the constant current, alternating electrical stimulus, an initial "burst" of neural impulses produced a strong signal of the change in neural state, such being demonstrated by the speed of the onset reaction. Due to adaptation effects occurring in the nerve with the continued application of the stimulus, a reduced nerve input remained to be modulated with stimulus offset, and such was reflected by the offset RTs being longer than the corresponding onset RTs. Further tests of this adaptation hypothesis are underway.

\section{References}

Adrian, E. D. The basis of sensation. London: Christophers, 1928.

Foulke, E. Communication by electrical stimulation of the skin. Final Progress Report, University of Louisville, March 1965, DA-49-193-MD-2525.

Hawkes, G. R. Cutaneous discrimination of electrical activity. Amer. J. Psychol., 1961, 74, 45-53.

Kenshalo, D. R., \& Nafe, J. P. A quantitative theory of feeling: 1960. Psychol. Rev., 1962, 69, 17-33.

Siegel, $\mathbf{S}$. Nonparametric statistics for the behavioral sciences. New York: McGraw-Hill, 1956

Woodrow, $\mathbf{H}$. R. Reactions to the cessation of stimuli and their nervous mechanism. Psychol. Rev., 1915, 22, 423-452.

\section{Note}

1. This research was supported by National Aeronautics and Space Administration grant No. NGR 18-002-007. 